\title{
Prognostic value of an inflammation-based score in patients undergoing pre-operative chemotherapy followed by surgery for esophageal cancer
}

\author{
HIROSHI MIYATA, MAKOTO YAMASAKI, YUKINORI KUROKAWA, SHUJI TAKIGUCHI, \\ KIYOKAZU NAKAJIMA, YOSHIYUKI FUJIWARA, MASAKI MORI and YUICHIRO DOKI \\ Department of Gastroenterological Surgery, Graduate School of Medicine, Osaka University, Osaka, Japan
}

Received April 21, 2011; Accepted June 7, 2011

DOI: $10.3892 /$ etm.2011.308

\begin{abstract}
Recent studies have shown that the presence of systemic inflammation is associated with poor outcome in patients with malignancy. However, whether systemic inflammation affects the response to pre-operative therapy and survival of patients undergoing multimodal treatment for esophageal cancer is not clear. We studied 152 patients who underwent pre-operative chemotherapy followed by surgery for esophageal cancer. The correlation between various clinicopathological factors, including hematological markers of systemic inflammatory response, and survival or response to chemotherapy was examined. Among various hematological factors, leucocyte count, hemoglobin level, albumin level, neutrophil-lymphocyte ratio and CEA, but not serum concentration of C-reactive protein, were significantly associated with survival. Multivariate analysis revealed that the clinical response to chemotherapy, number of metastatic lymph nodes, operative complications and systemic inflammation score (SI score), comprising leucocyte count, albumin and hemoglobin levels, were independent prognostic factors, and identified the SI score as the most significant prognostic factor. There was no significant relationship between hematological markers of systemic inflammation, including the SI score, and the response to chemotherapy. In conclusion, in patients scheduled for chemotherapy followed by surgery for esophageal cancer, systemic inflammation, reflected by SI, predicts poor outcome, but not the response to chemotherapy.
\end{abstract}

\section{Introduction}

Esophagectomy is the standard treatment for esophageal cancer. However, the majority of patients who undergo curative

Correspondence to: Dr Hiroshi Miyata, Department of Gastroenterological Surgery, Graduate School of Medicine, Osaka University, 2-2 Yamadaoka, Suita, Osaka 565-0871, Japan

E-mail: hmiyata@gesurg.med.osaka-u.ac.jp

Key words: esophageal cancer, chemotherapy, inflammation, albumin, hemoglobin resection subsequently develop local or systemic recurrence, and the 5-year survival rate ranges from 15 to $39 \%(1,2)$. To improve prognosis, the combination of peri-operative chemotherapy and/or radiotherapy is used in conjunction with esophagectomy. Neoadjuvant chemoradiotherapy followed by surgery is one of the most promising strategies for advanced esophageal cancers, and several studies have reported survival advantages of this trimodality therapy compared with surgery alone (3-5). Another potential combination therapy is neoadjuvant chemotherapy followed by surgery, and several studies have reported encouraging results (6-8). Thus, multimodal treatment combining surgery with other treatments has achieved mainstream status as a curative therapy for advanced esophageal cancer.

Recently, there is increasing evidence that a systemic inflammatory response is of prognostic value in patients with various types of cancers. Elevated serum C-reactive protein concentration is associated with poor prognosis in colorectal, breast and ovarian cancers (9-11). Another commonly used biomarker for systemic inflammation is serum albumin concentration. Low serum albumin concentration, often in combination with elevated C-reactive protein, is reported to be a predictor of poor prognosis in various types of cancers including colorectal cancers (12-14). In esophageal cancers, previous studies have also shown that serum C-reactive protein as a biomarker of systemic inflammation is useful for predicting the prognosis of patients undergoing esophagectomy (15-18). However, whether such serum biomarkers correlate with the outcome of pre-operative therapy and survival of patients undergoing multimodal treatment is not clear at present.

In the present study, we determined whether the presence of systemic inflammation predicts the outcome of pre-operative chemotherapy and survival of patients undergoing pre-operative chemotherapy followed by surgery for advanced esophageal cancers. Moreover, we also established a scoring system for systemic inflammation that reflects prognosis of the same patients.

\section{Materials and methods}

Patient population. From April 2000 to September 2008, 385 patients with thoracic esophageal cancers underwent 
surgery at the Department of Gastroenterological Surgery, Graduate School of Medicine, Osaka University. Among these, 152 patients who received pre-operative chemotherapy followed by surgery were enrolled in the present study. All patients were newly diagnosed and had received no prior treatment. Basically, during the same period, patients with any T (cT1-4) and lymph node involvement, including regional lymph nodes (N1) and distant lymph nodes (M1 lym) without distant organ metastasis received pre-operative chemotherapy followed by surgery. All patients were younger than 80 years of age; had adequate cardiac, hepatic, renal and bone marrow reserve; and could tolerate both the planned chemotherapy and the following surgical procedures.

In this study, all patients were staged before and after surgery according to the criteria of the International Union Against Cancer (UICC). Pre-treatment clinical staging was based on endoscopy, computed tomography (CT) scans of the neck, chest and the upper abdomen as continuous 5-mm slices, and positron emission tomography (PET) scan. Lymph nodes were diagnosed as metastasis-positive on CT scan when they were $>1.0 \mathrm{~cm}$ in maximum transverse diameter. Lymph nodes visible but $<1.0 \mathrm{~cm}$ on the long axis on CT scan were regarded as metastasis-positive only when focal prominent 18-fluorodeoxy glucose (FDG) uptake, compared with normal mediastinal activity, was detected on the PET scan. The study protocol was approved by the Human Ethics Review Committee of Osaka University School of Medicine.

Hematological examination. Routine laboratory tests for leucocyte, neutrophil and lymphocyte counts, hemoglobin, albumin, C-reactive protein, carcinoembryonic antigen (CEA) and squamous cell carcinoma (SCC) antigen were carried out before the commencement of pre-operative chemotherapy. The serum concentration of C-reactive protein was measured using latex immunonephelometry method (normal range, 0-0.2 mg/ dl). Serum levels of CEA and SCC antigen were measured using an enzyme immunoassay method. Serum levels $<5 \mathrm{ng} /$ $\mathrm{ml}$ for CEA and $<2 \mathrm{ng} / \mathrm{ml}$ for SCC antigen were considered normal in this assay, respectively.

The systemic inflammatory score (SI score) was estimated as follows; a high leucocyte count $(>9,500 \mu \mathrm{l})$, low serum albumin level ( $<3.5 \mathrm{~g} / \mathrm{dl})$ and low hemoglobin level $(<12.5 \mathrm{mg} /$ dl) was each allocated a score of 1 . SI score was calculated by adding up the scores of the leucocyte count, albumin and hemoglobin (range of SI score, 0-3).

Treatment protocol. Pre-operative chemotherapy administered at our hospital consisted of cisplatin, adriamycin and 5-fluorouracil (5-FU) (19). Cisplatin was administered at $70 \mathrm{mg} / \mathrm{m}^{2}$, adriamycin at $35 \mathrm{mg} / \mathrm{m}^{2}$ by rapid intravenous infusion on Day 1; and $5-\mathrm{FU}$ at $700 \mathrm{mg} / \mathrm{m}^{2}$ administered by continuous intravenous infusion on Day 1-7. Two courses of chemotherapy were used, separated by a 4-week interval. All patients underwent surgery 3 to 5 weeks after completion of pre-operative chemotherapy.

With regard to the type of surgery, 146 patients underwent transthoracic esophagectomy with two- or three-field lymphadenectomy while 6 patients underwent esophagectomy through trans-hiatal approach. Complete tumor resection (R0) was performed in 142 patients, while 10 patients had incomplete tumor resection with microscopic (R1) or macroscopic residual tumors (R2). Peri-operative complications were observed in 57 $(37.5 \%)$ of the 152 patients, including pneumonia/respiratory failure in 27 , anastomotic leakage in 17 , wound infection in 15 , recurrent nerve palsy in 15 , arrhythmia in 6 , ischemia related to gastric tube in 3 , chymothorax in 3 , bleeding in 3 , abdominal abscess in 2, cardiovascular failure in 3 and renal dysfunction in 2 .

Evaluation of response to chemotherapy. In order to evaluate the clinical response to chemotherapy, 2 weeks after completion of chemotherapy, all patients were re-staged by endoscopy, CT scan, and PET scan. The clinical response was categorized according to the World Health Organization response criteria for measurable disease and the criteria of the Japanese Society for Esophageal Diseases (20). A complete response (CR) was defined as clinical complete regression of the disease. A CR of the primary tumors represented complete disappearance of the tumors on CT scan and/or PET scan and endoscopy, and excluded those confirmed to have further ulceration on endoscopy and cancer cells in endoscopically obtained biopsy samples. A partial response (PR) was defined as $>50 \%$ reduction in primary tumor size and lymph node metastasis, as confirmed by CT scan. Progressive disease (PD) was defined as $>25 \%$ increase in the primary tumor or the appearance of new lesions. Cases that did not meet the criteria of PR or PD were defined as stable disease (SD).

The degree of histopathological tumor regression in surgical specimens was classified into 5 categories. The extent of viable residual carcinoma at the primary site was assessed semi-quantitatively, based on the estimated percentage of viable residual carcinoma in relation to the macroscopically identifiable tumor bed that was evaluated histopathologically. The percentage of viable residual tumor cells within the entire cancerous tissue was assessed as follows: grade 3 , no viable residual tumor cells; grade $2,<1 / 3$ residual tumor cells; grade $1 b, 1 / 3-2 / 3$ residual tumor cells; grade $1 \mathrm{a},>2 / 3$ residual tumor cells; grade 0 , no significant response to chemotherapy $(19,20)$. The extent of residual carcinoma in regional lymph nodes was not assessed.

Follow-up study. Following hospital discharge, patients were examined every 2 months for the first 2 years, and every 3 months thereafter. Computed tomography of the neck, thorax and upper abdomen was performed every 4 months for the first 2 years and every 6 months thereafter. Upper gastrointestinal endoscopy was performed annually. Recurrence was confirmed by CT scan and, when necessary, PET scan. All data were collected, entered prospectively into a database and updated at regular intervals. The median follow-up period of all 152 patients was 60.2 months (range, 20.1-120.8).

Statistical analysis. Overall survival was calculated from the date of surgery to the occurrence of the event or to the last known date of follow-up. Actual survival was calculated by the Kaplan-Meier method and statistically evaluated by the log-rank test. The Cox proportional hazards regression model was used to analyze the simultaneous influence of prognostic factors. The relationships between SI score and various clinicopathological factors were examined by the Mann-Whitney U 
Table I. Characteristics of the 152 patients who underwent preoperative chemotherapy followed by surgery for esophageal cancers.

\begin{tabular}{lr}
\hline Variable & $\mathrm{n}$ \\
\hline Age (years), mean \pm SD & $62.5 \pm 8.4$ \\
Gender & \\
Male & 132 \\
Female & 20 \\
Tumor location & \\
Upper third & 18 \\
Middle third & 63 \\
Lower third & 71 \\
Tumor depth & \\
cT1 & 6 \\
cT2 & 30 \\
cT3 & 84 \\
cT4 & 32 \\
Lymph node involvement & \\
cN0 & 9 \\
cN1 & 143 \\
Tumor stage & \\
cStage II & \\
cStage III & 30 \\
cStage IV & 77 \\
\hline
\end{tabular}

test. In all analyses, a $\mathrm{P}<0.05$ was accepted as statistically significant. These analyses were carried out using StatView J5.0 software package (Abacus Concepts, Inc., Berkeley, CA).

\section{Results}

Prognostic significance of systemic inflammatory response. Table I lists the characteristics of the patients who underwent pre-operative chemotherapy followed by surgery. The majority of patients were males and had squamous cell carcinoma with lymph node metastasis. Among various hematological factors including biomarkers for systemic inflammation such as C-reactive protein and albumin, we first identified predictors of prognosis of patients undergoing pre-operative chemotherapy. In this study, a C-reactive protein concentration $>1.0 \mathrm{mg} / \mathrm{dl}$ and albumin level $<3.5 \mathrm{mg} / \mathrm{dl}$ were considered to reflect the presence of systemic inflammation, based on previous studies (9,12-14). Upon univariate analysis, the leucocyte count, hemoglobin, albumin level and CEA were significantly associated with survival, while the serum concentration of C-reactive protein was not (Table II and Fig. 1). The neutrophil-lymphocyte ratio, which was reported previously to be a potential marker of inflammation $(21,22)$, was also a significant factor influencing patient survival. In addition to these hematological factors, univariate analysis showed that clinical tumor depth, clinical response to chemotherapy, pathological tumor depth, number of metastatic lymph nodes and operative complications were significantly correlated with prognosis. While leucocyte
Table II. Results of univariate analysis of overall survival.

Variable Patients HR $95 \%$ CI P-value

Pre-treatment factors

Age (years)

$(<70 / \geq 70)$

$118 / 34 \quad 1.01 \quad 0.60-1.71 \quad 0.9545$

Gender

(Female/male)

$20 / 132 \quad 1.04 \quad 0.55-1.96 \quad 0.9047$

Body mass index $\left(\mathrm{kg} / \mathrm{m}^{2}\right)$

$(\geq 18.5 /<18.5)$

$111 / 41 \quad 0.87 \quad 0.53-1.43 \quad 0.5811$

Tumor location

(Ut-Mt/Lt)

$81 / 71 \quad 1.20 \quad 0.79-1.83 \quad 0.3929$

Tumor depth

(cT1-2/cT3-4)

Lymph node involvement (cN0/cN1)

Histology

(SCC/others)

$36 / 116 \quad 1.82 \quad 1.04-3.20 \quad 0.0352$

$9 / 143 \quad 1.41 \quad 0.52-3.86 \quad 0.5001$

$\begin{array}{llll}140 / 12 & 0.89 & 0.38-2.06 & 0.7814\end{array}$

Hematological factors

Leucocyte count $(/ \mu 1)$

$(<9500 / \geq 9500)$

$128 / 24 \quad 1.87 \quad 1.07-3.25 \quad 0.0271$

Neutrophil count $(/ \mu 1)$

$(<6000 / \geq 6000)$

Lymphocyte count $(/ \mu 1)$

$(\geq 1500 /<1500)$

Neutrophil-lymphocyte ratio $(<4.0 / \geq 4.0)$

Hemoglobin $(\mathrm{mg} / \mathrm{dl})$

$(\geq 12.5 /<12.5)$

Albumin $(\mathrm{g} / \mathrm{dl})$

$(\geq 3.5 /<3.5)$

C-reactive protein $(\mathrm{mg} / \mathrm{dl})$

$(<1.0 / \geq 1.0)$

$111 / 41 \quad 1.32 \quad 0.82-2.12 \quad 0.2602$

$93 / 59 \quad 1.06 \quad 0.67-1.66 \quad 0.8164$

$107 / 45 \quad 1.63 \quad 1.03-2.58 \quad 0.0387$

$114 / 38 \quad 1.91 \quad 1.21-3.01 \quad 0.0056$

$102 / 50 \quad 2.03 \quad 1.33-3.12 \quad 0.0011$

$109 / 43 \quad 1.22 \quad 0.75-1.97 \quad 0.4207$

SI score

(0-1/2-3)

$123 / 29 \quad 2.72 \quad 1.68-4.42<0.0001$

$\mathrm{CEA}(\mathrm{ng} / \mathrm{ml})$

$(<5.0 / \geq 5.0)$

SCC antigen (ng/ml)

$(<2.0 / \geq 2.0)$

$\begin{array}{llll}125 / 27 & 1.87 & 1.10-3.17 & 0.0205\end{array}$

$131 / 21 \quad 1.20 \quad 0.65-2.24 \quad 0.5544$

Post-treatment factors

Tumor depth

(ypT1-2/ypT3-4)

$\begin{array}{llll}56 / 96 & 2.24 & 1.37-3.68 & 0.0013\end{array}$

No of metastatic lymph nodes

$(<3 / \geq 3)$

$91 / 61 \quad 3.13 \quad 2.02-4.85<0.0001$

Clinical response

(CR-PR/SD-PD)

$\begin{array}{llll}(83 / 69) & 1.72 & 1.12-2.63 & 0.0130\end{array}$

Pathological response

(major/minor response)

$24 / 128 \quad 1.13 \quad 0.63-2.05 \quad 0.6850$

Operative complication

(absent/present)

$\begin{array}{llll}95 / 57 & 1.82 & 1.19-2.79 & 0.0060\end{array}$

HR, hazard ratio; 95\% CI, 95\% confidence interval; Ut, upper third; Mt, middle third; Lt, lower third; SI score, systemic inflammation score; CEA, carcinoembryonic antigen; SCC, squamous cell carcinoma; CR, complete response; $\mathrm{PR}$, partial response; $\mathrm{SD}$, stable disease; $\mathrm{PD}$, progressive disease; major response, grade 2-3; minor response, grade $1 \mathrm{~b}-0$. 

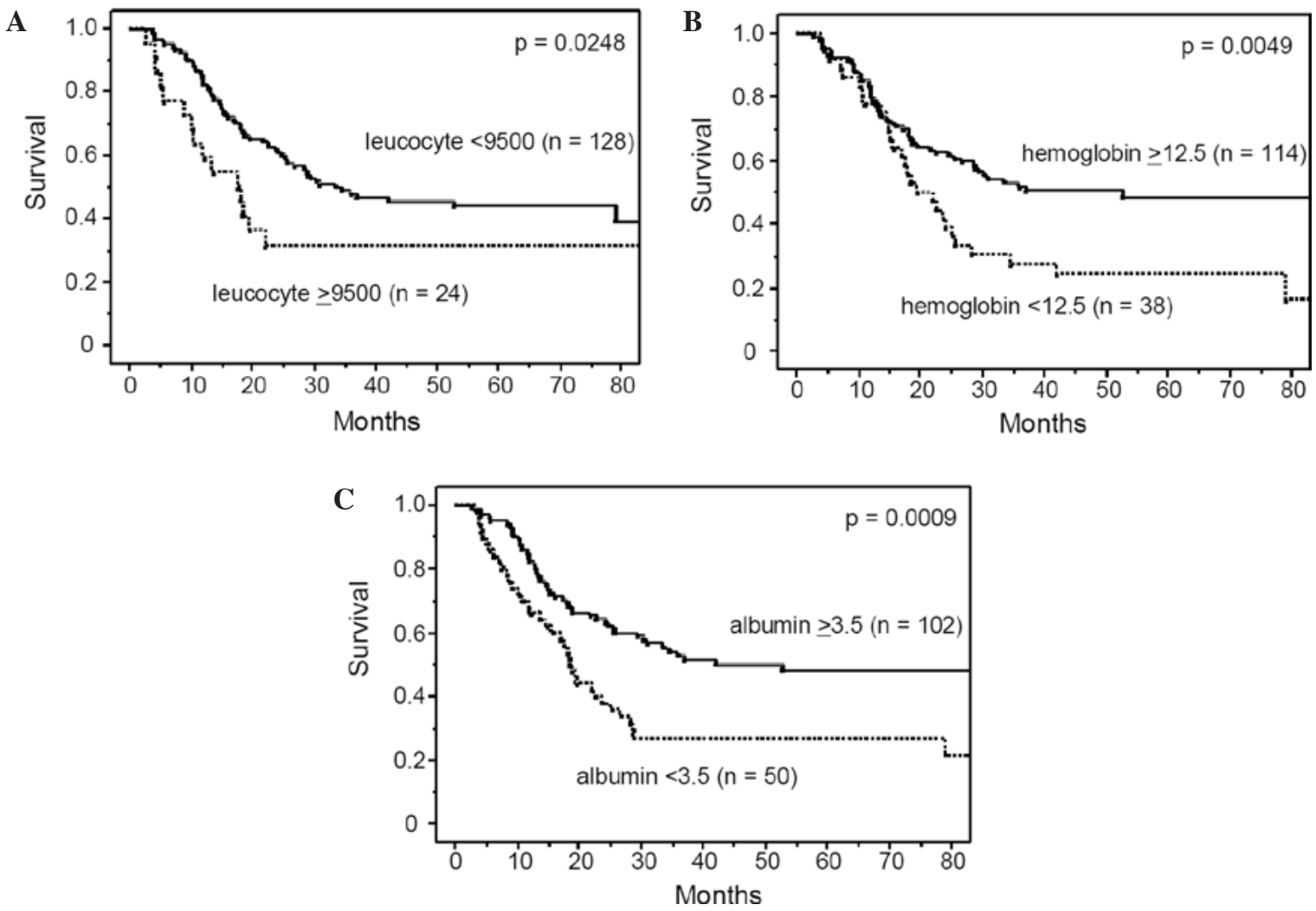

Figure 1. Overall survival rate of the 152 patients with esophageal cancers who received pre-operative chemotherapy followed by surgery, according to leucocyte count (A), hemoglobin level (B) and albumin level (C). The overall survival rates were significantly worse for patients with leucocytosis $(\geq 9500)$, anemia $(<12.5)$ and hypoalbuminemia $(<3.5)$.

Table III. Results of the multivariate analysis of overall survival.

\begin{tabular}{lllll}
\hline Variables & & HR & $95 \%$ CI & P-value \\
\hline Initial tumor depth & cT1-2 vs. cT3-4 & 0.78 & $0.42-1.45$ & 0.4463 \\
Clinical response & CR-PR vs. SD-PD & 1.78 & $1.08-2.94$ & 0.0245 \\
Neutrophil-lymphocyte ratio & $(<4.0$ vs. $\geq 4.0)$ & 1.30 & $0.76-2.22$ & 0.3362 \\
SI score & $(0-1$ vs. $2-3)$ & 3.17 & $1.74-5.78$ & 0.0002 \\
CEA $(\mathrm{ng} / \mathrm{ml})$ & $(<5.0$ vs. $\geq 5.0)$ & 1.31 & $0.75-2.49$ & 0.3362 \\
Pathological tumor depth & (ypT1-2 vs. ypT3-4) & 1.40 & $0.78-2.49$ & 0.2565 \\
No of metastatic lymph nodes & $(<3$ vs. $\geq 3)$ & 3.12 & $1.92-5.08$ & $<0.0001$ \\
Operative complication & (absent vs. present) & 1.73 & $1.09-2.75$ & 0.0204 \\
\hline
\end{tabular}

HR, hazard ratio; 95\% CI, 95\% confidence interval; SI score, systemic inflammation score; CEA, carcinoembryonic antigen; CR, complete response; PR, partial response; $\mathrm{SD}$, stable disease; $\mathrm{PD}$, progressive disease.

count and albumin are well-known markers of systemic inflammation, cancer-related anemia in patients with cancer is also reported to be a marker of cancer-related chronic inflammation (23-25). Therefore, we established a systemic inflammation score (SI score) using a high leucocyte count, low serum albumin and low hemoglobin level, all of which were identified as significant prognostic factors in our univariate analysis. Multivariate analysis identified the clinical response, SI score, number of metastatic lymph nodes and operative complications as significant and independent prognostic factors, and identified SI score as the most significant prognostic factor, along with the number of metastatic lymph nodes (Table III and Fig. 2).
Relationship between systemic inflammation and clinicopathological factors. Next, we examined the relationship between systemic inflammation (SI score) and various clinicopathological factors, including response to pre-operative chemotherapy. The SI score was significantly correlated with clinical tumor depth, serum concentration of C-reactive protein and SCC antigen level (Table IV). Furthermore, intraoperative complications were more frequent in patients with a high SI score than those with a lower SI score. However, there was no significant relationship between SI score and response to chemotherapy, although the latter was an independent prognostic factor. There was also no significant relationship 


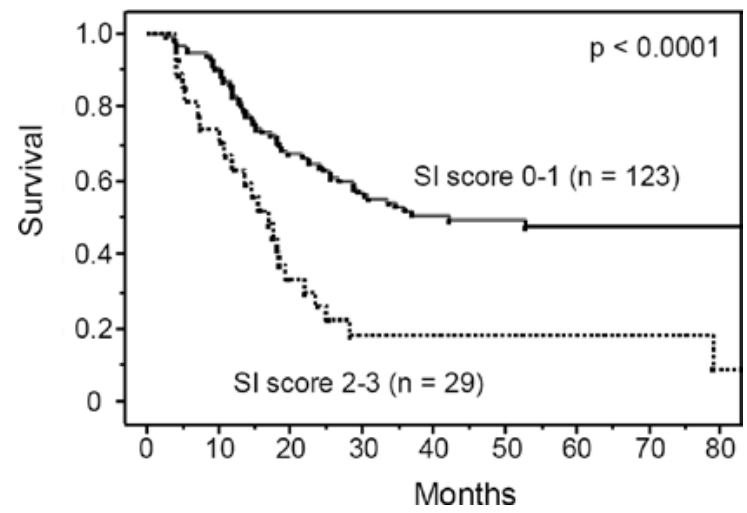

Figure 2. Overall survival rate of the 152 patients with esophageal cancers who received preoperative chemotherapy followed by surgery, according to the systemic inflammation score (SI score). The overall survival was significantly worse for patients with a high SI score (2-3), compared to patients with low SI score (0-1).

between response to chemotherapy and various hematological factors other than SI score, such as C-reactive protein and neutrophil/lymphocyte ratio.

\section{Discussion}

In the present study, we examined whether the presence of systemic inflammation affects prognosis and response to chemotherapy in patients undergoing pre-operative chemotherapy followed by surgery for advanced esophageal cancers. The results showed that the presence of systemic inflammation was associated with shortened survival of patients undergoing pre-operative chemotherapy but it had no significant influence on the response to chemotherapy. We also established a unique scoring system for systemic inflammation in patients undergoing multimodal treatment for esophageal cancers, which consisted of high leucocyte count, low serum albumin and low hemoglobin level. The SI score established in this study was identified as the most important prognostic factor on multivariate analysis.

In previous studies, the most commonly used biomarker of systemic inflammation in malignant disease was serum $\mathrm{C}$-reactive protein. C-reactive protein is produced in the liver and its production is regulated by pro-inflammatory cytokines such as interleukin (IL)-6, IL-1 and tumor necrosis factor (TNF) $\alpha$ (26-28). Several previous studies demonstrated that a pre-operative high C-reactive protein level was inversely correlated with survival of patients with esophageal cancer (15-18). In these studies, the cutoff value of C-reactive protein ranged from 0.5 to $1.0 \mathrm{mg} / \mathrm{dl}$. In this study, we attempted to use several cutoff values ranging from 0.5 to $1.0 \mathrm{mg} / \mathrm{dl}$, but a high C-reactive value did not predict the survival of patients undergoing pre-operative chemotherapy/surgery for esophageal cancers, irrespective of the cutoff value.

Another commonly used biomarker of systemic inflammation in malignancy is the serum albumin concentration. Hypoalbuminemia, which is often used as an indicator of poor nutrition, is also known to correlate with systemic inflammation through high levels of pro-inflammatory cytokines (29-31). In esophageal cancer, Ikeda et al (17) reported that
Table IV. Relationship between clinicopathological factors and SI score.

\begin{tabular}{|c|c|c|c|c|c|}
\hline \multirow[b]{2}{*}{ Variables } & \multirow[b]{2}{*}{$\mathrm{n}$} & \multicolumn{3}{|c|}{ SI score } & \multirow[b]{2}{*}{ P-value } \\
\hline & & 0 & 1 & $2-3$ & \\
\hline No. of patients & & 83 & 40 & 29 & \\
\hline \multicolumn{6}{|l|}{ Age (years) } \\
\hline$<70$ & 118 & 66 & 32 & 20 & 0.2480 \\
\hline$\geq 70$ & 34 & 17 & 8 & 9 & \\
\hline \multicolumn{6}{|l|}{ Gender } \\
\hline Male & 132 & 73 & 33 & 26 & 0.8511 \\
\hline Female & 20 & 10 & 7 & 3 & \\
\hline \multicolumn{6}{|l|}{$\mathrm{cT}$} \\
\hline cT1-2 & 36 & 27 & 6 & 3 & 0.0150 \\
\hline cT3-4 & 116 & 56 & 34 & 26 & \\
\hline \multicolumn{6}{|l|}{$\mathrm{cN}$} \\
\hline $\mathrm{cNO}$ & 9 & 5 & 2 & 2 & 0.4598 \\
\hline $\mathrm{cN} 1$ & 143 & 78 & 38 & 27 & \\
\hline \multicolumn{6}{|c|}{ C-reactive protein $(\mathrm{mg} / \mathrm{dl})$} \\
\hline$<1.0$ & 109 & 70 & 27 & 12 & 0.0002 \\
\hline$\geq 1.0$ & 43 & 13 & 13 & 17 & \\
\hline \multicolumn{6}{|c|}{ Neutrophil-lymphocyte ratio } \\
\hline$<4.0$ & 107 & 68 & 26 & 13 & 0.0004 \\
\hline$\geq 4.0$ & 45 & 15 & 14 & 16 & \\
\hline \multicolumn{6}{|l|}{ CEA (ng/ml) } \\
\hline$<5$ & 125 & 69 & 35 & 21 & 0.4271 \\
\hline$\geq 5$ & 27 & 14 & 5 & 8 & \\
\hline \multicolumn{6}{|c|}{ SCC antigen (ng/ml) } \\
\hline$<2$ & 131 & 78 & 37 & 16 & 0.0002 \\
\hline$\geq 2$ & 21 & 5 & 3 & 13 & \\
\hline \multicolumn{6}{|l|}{ Clinical response } \\
\hline CR-PR & 83 & 45 & 18 & 20 & 0.6487 \\
\hline SD-PD & 69 & 38 & 22 & 9 & \\
\hline \multicolumn{6}{|l|}{ Histopathology } \\
\hline $\mathrm{SCC}$ & 140 & 77 & 37 & 26 & 0.7984 \\
\hline Others & 12 & 6 & 3 & 3 & \\
\hline \multicolumn{6}{|l|}{$\mathrm{pT}$} \\
\hline pT1-2 & 56 & 33 & 12 & 11 & 0.7283 \\
\hline pT3-4 & 96 & 50 & 28 & 18 & \\
\hline \multicolumn{6}{|c|}{ No of metastatic lymph nodes } \\
\hline$<3$ & 61 & 32 & 20 & 9 & 0.7695 \\
\hline$\geq 3$ & 91 & 51 & 20 & 20 & \\
\hline \multicolumn{6}{|c|}{ Pathological response } \\
\hline Major response & 24 & 10 & 4 & 10 & 0.0571 \\
\hline Minor response & 128 & 73 & 36 & 19 & \\
\hline \multicolumn{6}{|c|}{ Operative complications } \\
\hline Absent & 95 & 58 & 23 & 14 & 0.0284 \\
\hline Present & 57 & 25 & 17 & 15 & \\
\hline
\end{tabular}

SI score, systemic inflammation score; CEA, carcinoembryonic antigen; SCC, squamous cell carcinoma; CR, complete response; PR, partial response; SD, stable disease; $\mathrm{PD}$, progressive disease; major response, grade 2-3; minor response, grade $1 \mathrm{~b}-0$. 
hypoalbuminemia was significantly correlated with poor survival of patients undergoing esophagectomy. Furthermore, recent studies also reported that hypoalbuminemia is predictive of the response to chemoradiotherapy and prognosis in patients treated by definitive chemoradiotherapy for esophageal cancers $(32,33)$. Hypoalbuminemia is sometimes used in combination with elevated C-reactive protein to calculate the inflammation-based prognostic score, Glasgow Prognostic score. The Glasgow Prognostic score is reported to be useful in predicting survival of patients with colorectal cancers, nonsmall cell lung cancers, pancreatic cancers and breast cancers (12-14). In esophageal cancers, Crumley et al (34) demonstrated that the Glasgow Prognostic score predicts cancer-specific survival of patients with inoperable gastroesophageal cancers. Kobayashi et al (35) also reported that the Glasgow Prognostic score determined before treatment is an independent predictor of survival of patients undergoing neoadjuvant chemoradiotherapy followed by surgery although their study was limited to 48 cases only. In the present study, the serum albumin level, but not Glasgow Prognostic score, was a marker of prognosis of patients undergoing pre-operative chemotherapy followed by surgery.

Anemia is also a marker of chronic inflammation. In patients with inflammatory disorders, pro-inflammatory cytokines, such as TNF $\alpha$, IL-1 and IFN- $\gamma$, do not only reduce the production of erythropoietin, which plays a crucial role in erythropoiesis, but also impair the response of erythroid progenitors to erythropoietin. In addition, the same pro-inflammatory cytokines can alter iron metabolism, leading to impaired erythropoiesis $(24,25)$. A previous study demonstrated that a low hemoglobin level was negatively correlated with serum levels of IL-6, IL-1 $\beta$, C-reactive protein and $\mathrm{TNF} \alpha$ in patients with ovarian cancer, suggesting that anemia in cancer is related to markers of inflammation (23). In the present study, a low hemoglobin level was associated with shorted survival of patients undergoing pre-operative chemotherapy for esophageal cancer, and we used a low hemoglobin level in addition to a low serum albumin concentration and high leucocyte count to estimate systemic inflammation. Thus, a low hemoglobin level is a potentially useful marker of systemic inflammation in patients undergoing multimodal treatment for advanced esophageal cancers.

The reason for our finding that systemic inflammation is not significantly associated with the response to chemotherapy but can predict survival of patients undergoing pre-operative chemotherapy followed by surgery is not clear. One possible explanation is that chronic inflammation may promote the spread of undetected cancer cells, micrometastasis, through polarization of M2 tumor-associated macrophages via cytokines, such as NF- $\mathrm{NB}$, and subsequent production of tumor growth factors or through the promotion of angiogenesis $(36,37)$. Another plausible explanation is that chronic inflammation may be associated with compromised immune function such as impaired T-lymphocytic response via various pro-inflammatory cytokines and chemokines $(38,39)$. In addition, this may lead to increased post-operative complications (40), which correlate with early cancer recurrence and poor prognosis $(41,42)$. Indeed, our study showed that systemic inflammation was significantly correlated with a high incidence of post-operative complications. Further studies are needed to examine the relationship between systemic inflammation and response to chemotherapy.
In summary, we established a scoring index for systemic inflammation (SI score) that comprises high leucocyte count, low serum albumin and low hemoglobin level, to predict poor survival of patients undergoing pre-operative chemotherapy followed by surgery for esophageal cancer. Our study also found no significant relationship between SI score and response to preoperative chemotherapy. However, the results showed that the SI score was the most important prognostic factor in patients undergoing multimodal treatment for esophageal cancer.

\section{References}

1. Muller JM, Erasmi H, Stelzner M, et al: Surgical therapy of oesophageal carcinoma. Br J Surg 77: 845-857, 1990.

2. Refaely Y and Krasna MJ: Multimodality therapy for esophageal cancer. Surg Clin North Am 82: 729-746, 2002.

3. Walsh TN, Noonan N, Hollywood D, et al: A comparison of multimodal therapy and surgery for esophageal adenocarcinoma. N Engl J Med 335: 462-467, 1996.

4. Gebski V,Burmeister B, Smithers BM, et al: Survival benefits from neoadjuvant chemoradiotherapy or chemotherapy in oesophageal carcinoma: a meta-analysis. Lancet Oncol 8: 226-234, 2007.

5. Tepper J, Krasna MJ, Niedzwiecki D, et al: Phase III trial of trimodality therapy with cisplatin, fluorouracil, radiotherapy, and surgery compared with surgery alone for esophageal cancer: CALGB 9781. J Clin Oncol 26: 1086-1092, 2008.

6. Law S, Fok M, Chow S, et al: Pre-operative chemotherapy versus surgical therapy alone for squamous cell carcinoma of the esophagus: a prospective randomized trial. J Thorac Cardiovasc Surg 114: 210-217, 1997.

7. Medical Research Council Oesophageal Cancer Working Group: Surgical resection with or without pre-operative chemotherapy in oesophageal cancer: a randomized controlled trial. Lancet 359: 1727-1733, 2002.

8. Yano M, Takachi K, Doki Y, et al: Pre-operative chemotherapy for clinically node-positive patients with squamous cell carcinoma of the esophagus. Dis Esophagus 19: 158-163, 2006.

9. McMillan Dc, Canna K and McArdle CS: Systemic inflammatory response predicts survival following curative resection of colorectal cancer. Br J Surg 90: 215-219, 2003.

10. Pierce BL, Ballard-Barbash R, Bernstein L, et al: Elevated biomarkers of inflammation are associated with reduced survival among breast cancer patients. J Clin Oncol 27: 3437-3444, 2009.

11. Kodama J, Miyagi Y, Seki N, et al: Serum C-reactive protein as a prognostic factor in patients with epithelial ovarian cancer. Eur J Obstet Gynecol Reprod Biol 82: 107-110, 1999.

12. Ishizuka M, Nagata $\mathbf{H}$, Takagi $\mathrm{K}$ and Kubota $\mathrm{K}$ : Influence of inflammation-based prognostic score on mortality of patients undergoing chemotherapy for far advanced or recurrent unresectable colorectal cancer. Ann Surg 250: 268-272, 2009.

13. Forrest LM, McMillan DC, McArdle CS, et al: A prospective longitudinal study of performance status, an inflammation-based score (GPS) and survival in patients with inoperable non-smallcell lung cancer. Br J Cancer 92: 1834-1836, 2005.

14. Al Murri AM, Bartlett JM, Canney PA, et al: Evaluation of an inflammation-based prognostic score (GPS) in patients with metastatic breast cancer. Br J Cancer 94: 227-230, 2006.

15. Nozoe T, Saeki H and Sugimachi K: Significance of pre-operative elevation of serum C-reactive protein as an indicator of prognosis in esophageal carcinoma. Am J Surg 182: 197-201, 2001.

16. Shimada H, Nabeya Y, Okazumi S, et al: Elevation of pre-operative serum C-reactive protein level is related to poor prognosis in esophageal squamous cell carcinoma. J Surg Oncol 83: 248-252, 2003.

17. Ikeda M, Natsugoe S, Ueno S, et al: Significant host- and tumorrelated factors for predicting prognosis in patients with esophageal carcinoma. Ann Surg 238: 197-202, 2003.

18. Zingg U, Forberger J, Rajcic B, et al: Association of C-reactive protein levels and long-term survival after neoadjuvant therapy and esophagectomy for esophageal cancer. J Gastrointest Surg 14: 462-469, 2010.

19. Miyata H, Yoshioka A, Yamasaki M, et al: Tumor budding in tumor invasive front predicts prognosis and survival of patients with esophageal squamous cell carcinomas receiving neoadjuvant chemotherapy. Cancer 115: 3324-3334, 2009. 
20. Japanese Society for Esophageal Diseases: Guidelines for the Clinical and Pathologic Studies on Carcinoma of the Esophagus. 10th edition. Kanehara Syuppan, Tokyo, 2007.

21. Gomez D, Farid S, Malik HZ, et al: Pre-operative neutrophil-tolymphocyte ratio as a prognostic predictor after curative resection for hepatocellular carcinoma. World J Surg 32: 1757-1762, 2008.

22. Walsh SR, Cook EJ, Goulder F, et al: Neutrophil-lymphocyte ratio as a prognostic factor in colorectal cancer. J Surg Oncol 91: $181-184,2005$

23. Macciò A, Madeddu C, Massa D, et al: Hemoglobin levels correlate with interleukin-6 levels in patients with advanced untreated epithelial ovarian cancer: role of inflammation in cancer-related anemia. Blood 106: 362-367, 2005.

24. Buck I, Morceau F, Grigorakaki C, et al: Linking anemia to inflammation and cancer: the crucial role of TNF alpha. Biochem Pharmacol 77: 1572-1579, 2009

25. Adamson JW: The anemia of inflammation/malignancy: mechanisms and management. Hematology Am Soc Hematol Educ Program 2008: 159-165, 2008

26. Gelin J, Moldawer LL, Lönnroth C, et al: Role of endogenous tumor necrosis factor alpha and interleukin 1 for experimental tumor growth and the development of cancer cachexia. Cancer Res 51: 415-421, 1991.

27. Heikkilä K, Ebrahim S and Lawlor DA: A systematic review of the association between circulating concentrations of $\mathrm{C}$ reactive protein and cancer. J Epidemiol Community Health 61: 824-833, 2007.

28. Coussens LM and Werb Z: Inflammation and cancer. Nature 420 : 860-867, 2002

29. McMillan DC, Watson WS, O'Gorman P, et al: Albumin concentrations are primarily determined by the body cell mass and the systemic inflammatory response in cancer patients with weight loss. Nutr Cancer 39: 210-213, 2001.

30. Argilés JM, Busquets S and López-Soriano FJ: Cytokines as mediators and targets for cancer cachexia. Cancer Treat Res 130: 199-217, 2006

31. Neal CP, Mann CD, Sutton CD, et al: Evaluation of the prognostic value of systemic inflammation and socioeconomic deprivation in patients with resectable colorectal liver metastases. Eur J Cancer 45: 56-64, 2009.

32. Wang CY, Hsieh MJ, Chiu YC, et al: Higher serum C-reactive protein concentration and hypoalbuminemia are poor prognostic indicators in patients with esophageal cancer undergoing radiotherapy. Radiother Oncol 92: 270-275, 2009.
33. Di Fiore F, Lecleire S, Pop D, et al: Baseline nutritional status is predictive of response to treatment and survival in patients treated by definitive chemoradiotherapy for a locally advanced esophageal cancer. Am J Gastroenterol 102: 2557-2563, 2007.

34. Crumley AB, McMillan DC, McKernan M, et al: Evaluation of an inflammation-based prognostic score in patients with inoperable gastro-oesophageal cancer. Br J Cancer 94: 637-641, 2006.

35. Kobayashi T, Teruya M, Kishiki T, et al: Inflammation-based prognostic score, prior to neoadjuvant chemoradiotherapy, predicts postoperative outcome in patients with esophageal squamous cell carcinoma. Surgery 144: 729-735, 2008.

36. DeNardo DG and Coussens LM: Inflammation and breast cancer. Balancing immune response: crosstalk between adaptive and innate immune cells during breast cancer progression. Breast Cancer Res 9: 212, 2007.

37. Ulrich CM, Bigler J and Potter JD: Non-steroidal anti-inflammatory drugs for cancer prevention: promise, perils and pharmacogenetics. Nat Rev Cancer 6: 130-140, 2006.

38. Macciò A, Lai P, Santona MC, et al: High serum levels of soluble IL-2 receptor, cytokines, and C reactive protein correlate with impairment of $\mathrm{T}$ cell response in patients with advanced epithelial ovarian cancer. Gynecol Oncol 69: 248-252, 1998.

39. Canna K, McArdle PA, McMillan DC, et al: The relationship between tumour T-lymphocyte infiltration, the systemic inflammatory response and survival in patients undergoing curative resection for colorectal cancer. Br J Cancer 92: 651-654, 2005.

40. Moyes LH, Leitch EF, McKee RF, et al: Pre-operative systemic inflammation predicts postoperative infectious complications in patients undergoing curative resection for colorectal cancer. $\mathrm{Br} J$ Cancer 100: 1236-1239, 2009.

41. Lerut T, Moons J, Coosemans W, et al: Postoperative complications after transthoracic esophagectomy for cancer of the esophagus and gastroesophageal junction are correlated with early cancer recurrence: role of systematic grading of complications using the modified Clavien classification. Ann Surg 250: 798-807, 2009.

42. Lagarde SM, de Boer JD, ten Kate FJ, et al: Postoperative complications after esophagectomy for adenocarcinoma of the esophagus are related to timing of death due to recurrence. Ann Surg 247: 71-76, 2008 